\title{
Shade Interest Points for Dynamic Stream Object Categorization
}

\author{
S. Suresh Babu ${ }^{1}$, Venkata Naresh Mandhala ${ }^{1}$, Siva Koteswara Rao Chinnam ${ }^{1}$ and \\ Tai-hoon Kim ${ }^{2}$ \\ ${ }^{1}$ Information Technology Department, \\ VFSTR University, \\ Vadlamudi-522213, Guntur, India \\ ${ }^{2}$ Department of Convergence Security, \\ Sungshin Women's University, \\ 249-1, Dongseon-dong 3-ga, \\ Seoul, 136-742, Korea \\ mvnaresh.mca@gmail.com ${ }^{1}$,taihoonn@daum.net ${ }^{2}$ (Corresponding Author)
}

\begin{abstract}
Discovery of investment focuses for ensuing handling is one of the fundamental parts of machine vision. Object order of pictures vigorously depends on investment point identification from which nearby picture descriptors are registered for picture matching. Since investment focuses are focused around luminance, past methodologies generally overlooked the color viewpoint. Later an approach that uses saliency-based peculiarity determination improved by a primary part dissection based scale choice strategy is created. It is utilized to lessen the affectability to changing imaging conditions, and hence it is a light-invariant investment point's location framework. Utilization of color expands the uniqueness of investment focuses. In the setting of item distinguishment, the human observation framework is regularly pulled in by contrasts between parts of pictures and by movement or moving articles. In this manner, in the feature indexing system, investment focuses give more helpful data when contrasted with static pictures. So we propose to amplify the above methodology for element feature streams utilizing SpaceTime Interest Points (Stips) that uses a calculation for scale adaption of spatio-worldly investment focuses. STIP distinguishes moving questions in features and describes some particular changes in the development of these articles. A handy execution of the proposed framework accepts our case to help element streams and further it could be utilized as a part of uses, for example, Motion Tracking, Entity Detection and Naming applications.
\end{abstract}

Keywords: Interest point calculation, Space-Time Interest Points, Machine Vision

\section{Introduction}

The distinguishment of surface and article classes is a standout amongst the most difficult issues in machine vision. Representation, identification and learning are the fundamental issues that need to be handled in outlining a visual framework for perceiving article classifications. Investment point recognition is an imperative exploration region in the field of picture transforming and machine vision. Picture recovery and article arrangement vigorously depend on investment point location from which nearby picture descriptors are figured for picture and item matching. Shade assumes an imperative part in the pre-attentive stage in which peculiarities are identified as it is one of the basic boost characteristics. It is standard to characterize composition as a visual example portrayed by the redundancy of a couple of fundamental primitives. There is wide concession to the issue of representation: item classifications are spoken to as gathering of gimmicks, each one section has a different appearance and spatial position. 
The current pattern in item distinguishment is to expanding the quantity of focuses applying a few locators or consolidating them or making the investment point dispersion as thick as could be expected under the circumstances. With the unstable development of picture and feature information sets, bunching and logged off preparing of gimmicks get to be less attainable. By decreasing the quantity of peculiarities and working with an anticipated number of meager gimmicks, bigger picture information sets could be handled in less time. A stable number of peculiarities lead to a more unsurprising workload for such errands. Late work has expected to discover unique gimmicks by performing an assessment of all peculiarities inside the information set or for every picture class and picking the most incessant ones. This methodology requires an extra estimation venture with an innate request on memory and handling time reliant on the quantity of gimmicks.

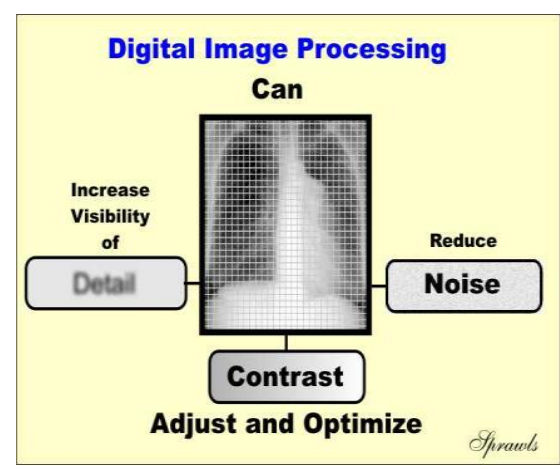

Figure 1. Characteristics of the Image Processing Operations

This option might along these lines give specific pursuit to vigorous peculiarities decreasing the aggregate number of investment focuses utilized for picture recovery. We propose color investment focuses to get a meager picture representation. Thus, we diminish the affectability to imaging conditions, light-invariant investment focuses are proposed. For shade helped focuses, the point is to endeavor color detail determined from the event likelihood of shades. Shade helped focuses are gotten through saliency-based peculiarity determination. The utilization of shade data permits separating repeatable and scale-invariant investment focuses. Color subordinates were taken to structure the premise of a shade saliency boosting capacity to equivalent the data substance and saliency of a given shade event. Our point is to choose investment focuses focused around shade discriminative and invariant properties determined from neighborhood neighborhoods. Our center is on shade demonstrates that have valuable perceptual and invariant properties to attain a decrease in the quantity of investment focuses. A technique for selecting a scale connected with the registered investment focuses while keeping up the properties of the shade space utilized and to direct the trademark scale by the saliency of the encompassed structure.

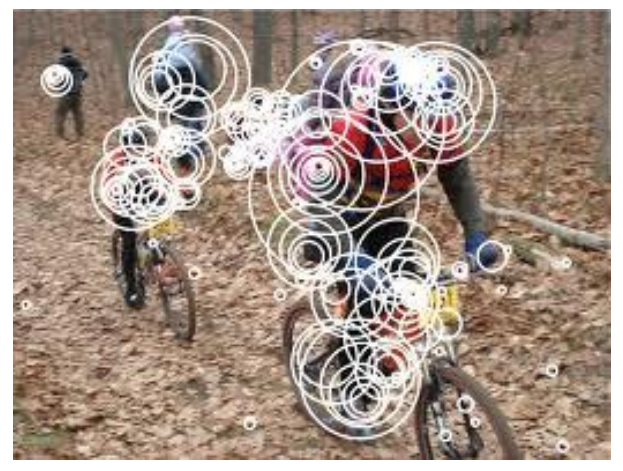

Figure 2. Image Interest Point calculation Event Mechanism 


\section{Background Work}

The primary steps of picture recovery and item arrangement are delineated. Regular Pipeline for Image Retrieval and Object Categorization: Characteristic extraction is completed with either worldwide or nearby gimmicks. Worldwide peculiarities need vigor against impediments and jumbling and give a quick and productive method for picture representation. The nearby peculiarities are either power or shade based investment focuses. Thick testing of neighborhood peculiarities has been utilized as it gives great execution. Descriptors speak to the nearby picture data around the investment focuses. This might be classified into three classes:

1) Distribution of certain neighborhood properties of the picture

Ex: Scale-invariant gimmick change

2) Spatial recurrence

Ex: Wavelets

3) Other differentials

Ex: Neighborhood planes

Productive approaches to figure these descriptors exist long ago ascertained results could be utilized. Bunching for mark era or vocabulary estimation doles out the descriptors into a subset of classifications. Because of the unreasonable memory and runtime prerequisites of progressive grouping, apportioned bunching is the system for decision in making peculiarity marks. Picture descriptors are contrasted and formerly learnt and put away models.

Characterization methodologies need peculiarity determination to toss insignificant and repetitive data. It is demonstrated that a capable matching step can effectively toss unessential data and better execution is picked up. A color Gaussian pyramid is utilized to prompts different questionable peculiarities and the powerlessness to match pictures at diverse scales. The strategy is free of the color space utilized. The subsidiaries of the invariants are joined in the Harris second minute grid. It uses settled scales for matching of pictures under fluctuating lighting.

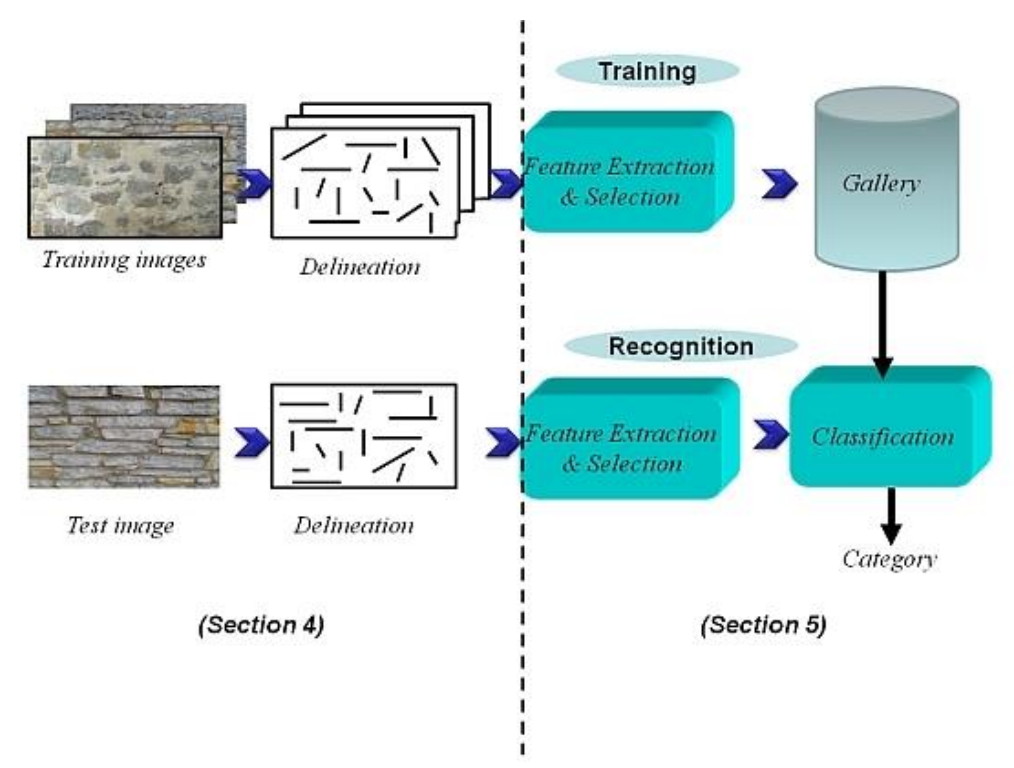

\section{Figure 3. Object Categorization in Image Processing Application Development}

A photometric semi invariant HIS shade space giving a corner indicator better commotion solidness attributes contrasted with existing photometric invariants and a color boosting speculation for characterizing striking colors. Our commitment is to 
augment this methodology by fusing a scale choice technique to identify shade investment for identification.

\section{Proposed Approach}

An article model comprises of various parts. Each one section has an appearance, relative scale and could be blocked or not. The shape is spoken to by the common position of the parts. Whole model is generative and probabilistic shape and impediments are all demonstrated by likelihood thickness capacities.

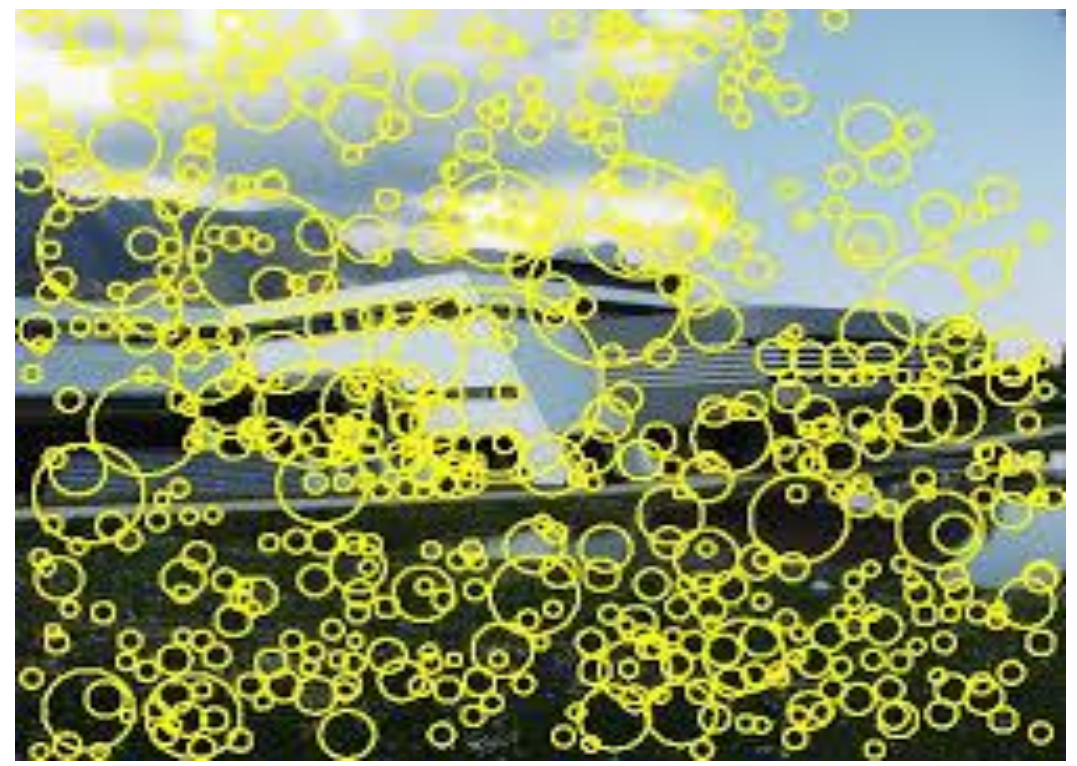

\section{Figure 4. Space Time Interest Point Calculation Process in Image retrieval}

Distinguishment is performed on a question picture by again first identifying districts and their scales and afterward assessing the areas in a Bayesian way. Peculiarities are discovered utilizing the indicator of Kadir and Brady.

The peculiarity finder distinguishes districts of enthusiasm on each one picture. Once the districts are distinguished, they are trimmed from the picture and rescaled to the extent of a little pixel patch. Each one patch exists in a 121 dimensional space. We should some way or another diminish the dimensionality of each one patch whilst holding its peculiarity. A 121-dimensional Gaussian is unmanageable from a numerical perspective furthermore the quantity of parameters included is such a large number of it would be impossible be evaluated. In the learning stage, we gather the patches from all pictures and perform PCA on them. Patch's appearance is then a vector of the directions inside the first key segments. This gives a decent remaking of the first fix whilst utilizing a moderate number of parameters for every part. Learning is done utilizing the desire amplification (EM) calculation which iteratively merges from some arbitrary beginning estimation of $\theta$ to a greatest. The scale data from each one peculiarity permits us to take in the model shape in a scale-invariant space. Learning complex models, for example, these has specific troubles. Shockingly, we expect given the unpredictability of the inquiry space, the calculation is surprising reliable in its joining. Beginning conditions were picked arbitrarily inside a sensible extend and union generally happened inside 50-100 EM emphasess. Evaluating this from closer view information demonstrated incorrect so the parameters were evaluated from a set of foundation pictures and not overhauled inside the EM emphasis. Distinguishment moves ahead by first identifying gimmicks and after that assessing these 
peculiarities utilizing the learnt model. By computing the probability proportion and contrasting it with an edge, the vicinity or nonappearance of the article inside the picture may be resolved. As in learning productive inquiry methods are utilized since substantial mean around 2-3 seconds are taken for every picture.

\section{Performance Evaluation}

Investigations were completed as takes after: every dataset was part haphazardly into two different sets of equivalent size. The choice was a straightforward item present/missing one, aside from the autos dataset where various occurrences of the article were to be found. A constrained measure of preprocessing was performed on a portion of the datasets. The spotted feline dataset was just 100 pictures initially and an alternate 100 were included by reflecting the first pictures making 200 altogether. There were two periods of investigations. Datasets with scale variability were standardized so the articles were of uniform size. Calculation was then assessed on the datasets and contrasted with different methodologies. The calculation was run on the datasets containing scale variety and the execution contrasted with the scale-standardized case. The main parameter that was balanced at all in all the accompanying investigations was the scale over which gimmicks were found. The face and motorbike datasets have tight shape demonstrates yet a percentage of the parts have a profoundly variable appearance. These parts any gimmick in that area will do paying little mind to what it would seem that. The larger part of mistakes are an aftereffect of the article accepting lacking scope from the peculiarity locator. One plausibility is that the edge is forced on $\mathrm{N}$ numerous gimmicks on the article are evacuated. The gimmick indicator appears to perform severely when the article is much darker than the foundation. The bunching of remarkable focuses into peculiarities inside the peculiarity finder. A review accuracy curve 1 (RPC) and a table contrasting the calculation with past methodologies to protest class distinguishment as indicated in the Figure 5.

\begin{tabular}{|c||c|c|c|}
\hline Dataset & Ours & Others & Ref. \\
\hline \hline Motorbikes & 92.5 & 84 & {$[17]$} \\
\hline Faces & 96.4 & 94 & {$[19]$} \\
\hline Airplanes & 90.2 & 68 & {$[17]$} \\
\hline Cars(Side) & 88.5 & 79 & {$[1]$} \\
\hline
\end{tabular}

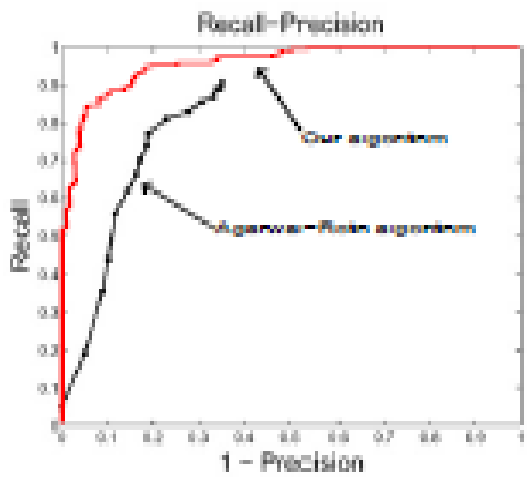

Figure 5. Comparison of Methods in Application Development

The outline on the right demonstrates the RPC for and our calculation on the autos dataset and on left the table gives ROC meet lapse rates on various datasets. The Figure 3 talk about the 6 normal face models. The upper left figure demonstrates the shape model. Circles speak to the change of each one section and the likelihood of each one section being available is demonstrated just to the right of the mean. Upper right figure demonstrates 10 patches closest to the mean of the appearance thickness for each one section and the foundation thickness. Alongside the determinant of the difference framework, to give a thought as to the relative snugness of every dissemination. The pink dabs are gimmicks found on each one picture and the hued rings demonstrate the peculiarities of the best theory in the picture. 

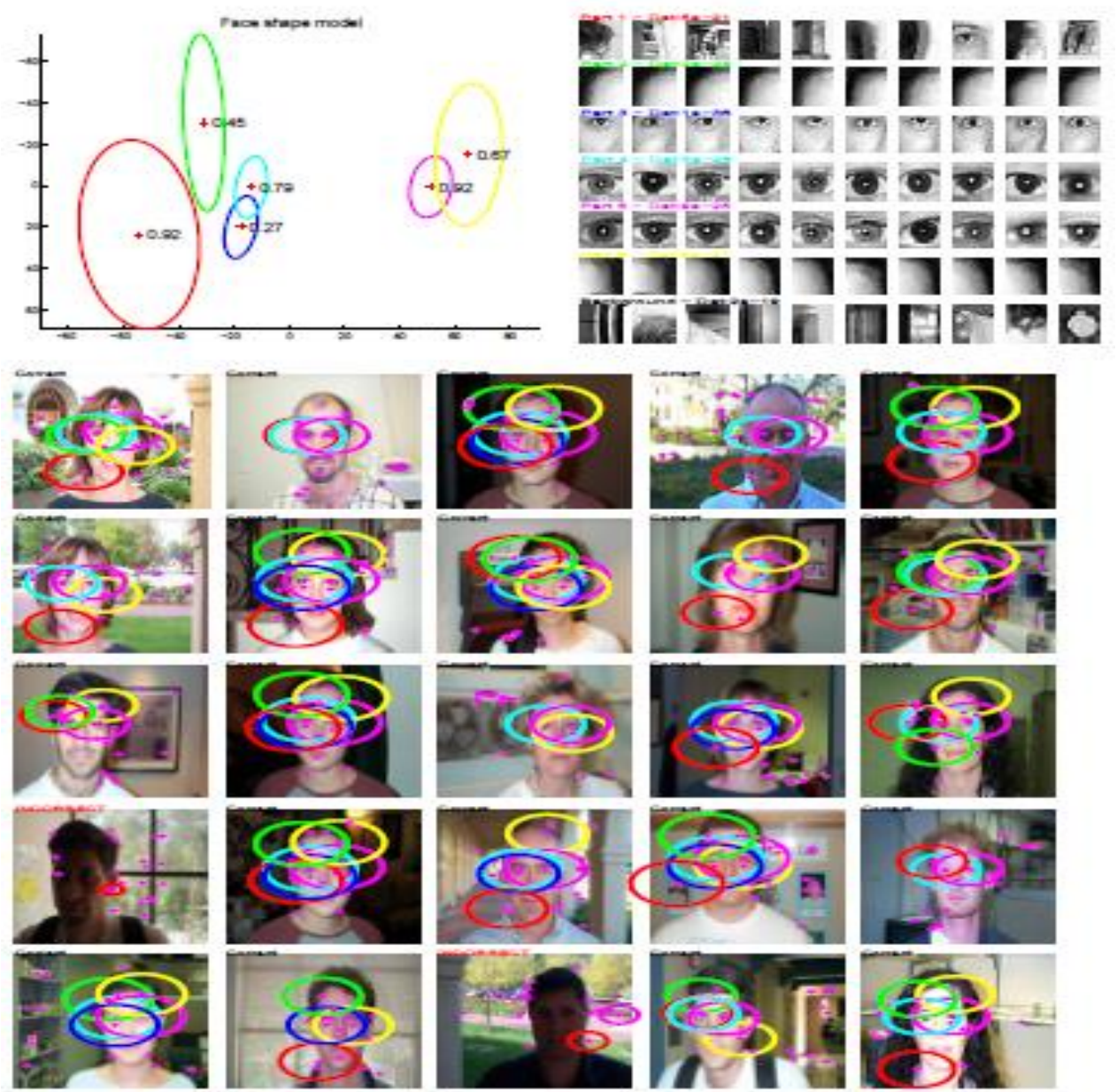

Figure 6. A Typical Face Model with 6 Parts

Size of the circles indicates the score of the hypothesis. Exactly the same algorithm settings are used for next consider example. As we consider the typical airplane with 6 parts.

The corner to corner demonstrates the ROC measure up to blunder rates on test information over four classifications that the calculation's parameters were kept precisely the same. The execution above could be enhanced significantly (planes to $94.0 \%$ and countenances to $96.8 \%$ ) if peculiarity scale is balanced on a for every dataset premise. The table demonstrates the execution of the calculation over the four datasets. The table demonstrates the perplexity between models which is normally at the level of possibility. The execution of the scale-invariant models on un-scaled pictures to that of the scalevariation shows on the pre-scaled information. There is a huge change in execution with the scale invariant model. Because of the gimmick finder performing severely on little pictures and in the pre-scaled case. This dataset was tried against foundation street scenes than the foundation pictures. 


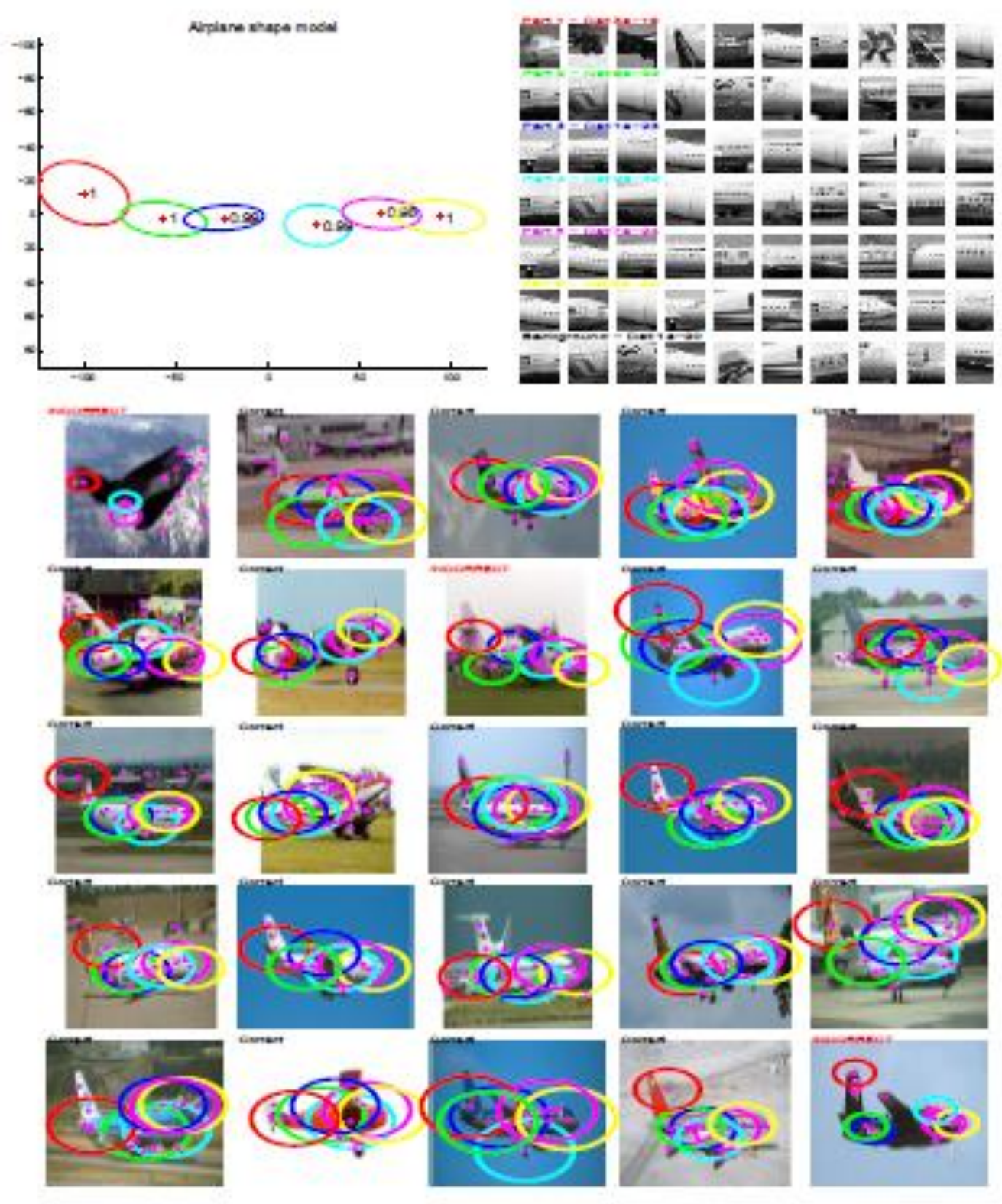

Figure 7. A Typical Airplane Model with 6 Parts

\section{Conclusion}

Investment point recognition is a paramount exploration zone in the field of picture preparing and machine vision. Its use could be found in the facial distinguishment, movement identification, permit plate location applications. To diminish the affectability to imaging conditions, light-invariant investment focuses are proposed. Characteristic determination happens at the first venture of peculiarity extraction and is completed freely for every gimmick. Former methodologies utilizing HSI, PCA blending plans focused on actualizing investment focuses order for item arrangement in shaded pictures. We propose to amplify the methodology for element feature streams utilizing Space-Time Interest Points (Stips) that uses a calculation for scale adaption of spatio-fleeting investment focuses. A down to earth usage of the proposed framework approves our case to help element streams and further it might be utilized within applications, for example, Motion Tracking, Entity Detection and Naming applications. 


\section{References}

[1] J. Stottinger, A. Hanbury, N. Sebe and T. Gevers, "Sparse colorinterest points for image retrieval and object categorization", IEEE Trans. Image Process, vol. 21, no. 5, (2012), pp. 2681 -2692.

[2] S. Agarwal and D. Roth, "Learning a sparse representation for object detection", In: Heyden, A., Sparr, G., Nielsen, M., Johansen, P. (eds.) ECCV 2002. LNCS, Springer, Heidelberg, vol. 2353, pp. 113-130.

[3] A. Yali and D. Geman, "A computational model for visual selection", Neural computation, (1999), vol. 11, no. 7, pp. 1691-1715.

[4] E. Borenstein and S. Ullman, "Class-specific, top-down segmentation”, In Proc. ECCV, (2002), pp. 109124.

[5] M. Burl, M. Weber and P. Perona, "A probabilistic approach to object recognition using local photometry and global geometry", In Proc. ECCV, (1998), pp. 628-641.

[6] R. Fergus, P. Perona, and A. Zisserman, "Object Class Recognition by Unsupervised Scale-Invariant Learning", In Proc. CVPR 2003, vol. 2, (2003), pp. 264-271.

[7] C. Harris and M. Stephens, "A combined corner and edge detection", in Proc. 4th Alvey Vis. Conf, (1988), pp. 147-151.

[8] T. Kadir and M. Brady, "Saliency, scale and image description", Int. J. Comput. Vis., vol. 45, no. 2, (2001) November pp. 83-105.

[9] K. Mikolajczyk and C. Schmid, "Scale and affine invariant interest point detectors", Int. J. Comput. Vis., vol. 60, no. 1, (2004) October, pp. 63-86. 